Supporting Information

for

\title{
Palladium-Catalyzed Oxidative Amination on Alkenes: Improved Catalyst Reoxidation Enables the Use of Alkene as the Limiting Reagent
}

Michelle M. Rogers, Vasily Kotov, Jaruwan Chatwichien and Shannon S. Stahl University of Wisconsin - Madison, 1101 University Ave.

Madison, WI 53706 


\section{General Considerations}

All commercially available compounds were used as received, and all were purchased from Aldrich, TCI America, Lancaster Synthesis except Palladium that was donated by Eli Lilly.

${ }^{1} \mathrm{H}$, and ${ }^{13} \mathrm{C}$ spectra were recorded on a Bruker AC-300 MHz, Varian Mercury-300 MHz, Varian Unity-500 MHz or Varian Inova-500 MHz spectrometers, and CDCl3 was purchased from Aldrich. The chemical shifts $(\delta)$ are given in parts per million relative to internal TMS $\left(0\right.$ ppm for $\left.{ }^{1} \mathrm{H}\right), \mathrm{CDCl}_{3}\left(77.24 \mathrm{ppm}\right.$ for $\left.{ }^{13} \mathrm{C}\right)$.

Flash column chromatography was performed on silica gel 60 (particle size 0.040-0.063mm, 230-400 mesh ASTM, purchased from Silicycle) with hexanes/ether or hexanes/ethyl acetate.

\section{General Procedure for Catalyst Screening.}

In a disposable culture tube, Palladium complexes $(0.018 \mathrm{mmol})$, Copper complexes $(0.018$ mmol $)$, additive $(0.0375$ $-0.375 \mathrm{mmol})$ and Phthalmide $(0.45 \mathrm{mmol})$ were combined. Reaction tubes were placed in a 48 -well parallel reactor mounted on a Large Capacity Mixer (Glas-Col) and the headspace was purged with molecular oxygen for ca. 20 min. A benzonitrile solution containing octadecane $(0.40 \mathrm{~mL})$ was added and the reactor was warmed to $60{ }^{\circ} \mathrm{C}$. After warming 1-tetradecene $(0.375 \mathrm{mmol}, 0.095 \mathrm{~mL})$ was added to each tube to initiate the reaction. The reactions were vortexed for 24 hours under 1 atm of molecular oxygen. After the reactions were stopped, 1,3,5-

trimethoxybenzene ( $1 \mathrm{~mL}$ of a known concentration solution in 1D-chloroform) was added to the reaction mixture. Samples were evaluated by GC and NMR for the products and remaining starting materials.

\section{Preparation of Alkenes with Protected Alcohols.}

The alkenes employed in Table 2, entries 10 and15-19 were prepared according to literature precedent. ${ }^{1,2}$ Characterization data for these compounds agreed with the literature reportes. ${ }^{1,3,4,5,6,7,8}$

\section{General Procedure for Palladium-Catalyzed Intermolecular Oxidative Amination.}

Into an Ace Glass heavy walled pressure tube (rated to $120 \mathrm{psig})$ was weighed $\mathrm{Pd}(\mathrm{OAc})_{2}(22.4 \mathrm{mg}, 0.1 \mathrm{mmol})$ and the nucleophile $(1.2 \mathrm{mmol})$. The reaction tube was connected to a custom swagelock reactor system. Benzonitrile $(1.33 \mathrm{~mL}, 0.75 \mathrm{M})$ was added. The reactor was pressurized to $20 \mathrm{psig}$ and heated to $60{ }^{\circ} \mathrm{C}$. Once the reactor had reached temperature, the alkene $(1.0 \mathrm{mmol})$ was added via syringe and the reactor was then pressurized to $60 \mathrm{psig}$. The reaction was stirred to 36-40 hours. The benzonitrile solvent was removed in vacuo, and the product was purified by column chromatography.

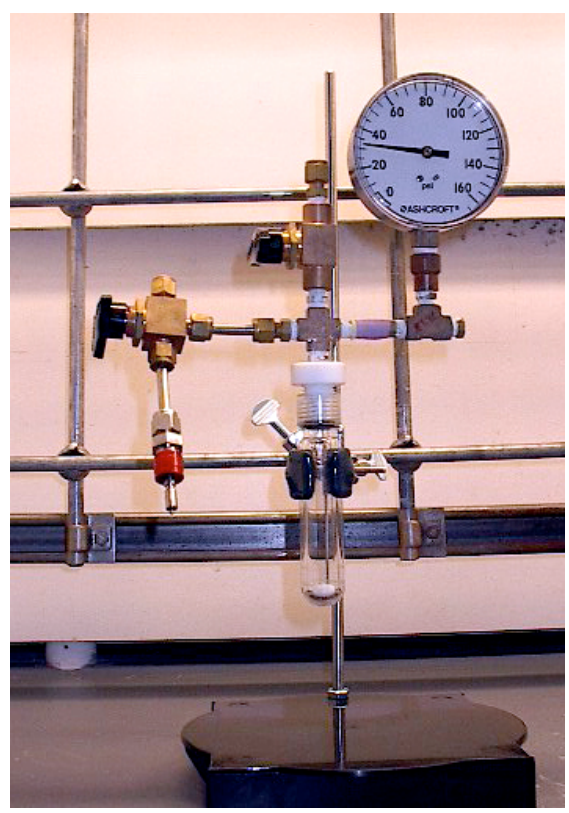


Product Characterization Data.

2-(1-Tridecyl-vinyl)-isoindole-1,3-dione. Column chromatography (hexanes/ether, 4:1) afforded a white solid, 72\% (227 mg). Major Isomer: ${ }^{1} \mathrm{H}$ NMR $\left(300 \mathrm{MHz}, \mathrm{CDCl}_{3}\right) \delta$ 7.89-7.84 (m, 2H), 7.75-7.72 (m, 2H), $5.38(\mathrm{t}, \mathrm{J}=1.2 \mathrm{~Hz}$, $1 \mathrm{H}), 5.13(\mathrm{~s}, 1 \mathrm{H}), 2.45(\mathrm{t}, \mathrm{J}=2.9 \mathrm{~Hz}, 2 \mathrm{H}), 1.43-1.23(\mathrm{~m}, 20 \mathrm{H}), 0.87(\mathrm{t}, \mathrm{J}=6.9 \mathrm{~Hz}, 3 \mathrm{H})$; Minor Isomer (integrations relative to the major isomer): ${ }^{1} \mathrm{H} \mathrm{NMR}\left(300 \mathrm{MHz}, \mathrm{CDCl}_{3}\right) \delta 5.52$ (tq, $\left.\mathrm{J}=7.5,1.2 \mathrm{~Hz}, .15 \mathrm{H}\right), 2.21(\mathrm{q}, \mathrm{J}=7.2 \mathrm{~Hz}$, $.41 \mathrm{H}), 1.96(\mathrm{~d}, \mathrm{~J}=1.2 \mathrm{~Hz}, 0.62 \mathrm{H})$; Major Isomer: ${ }^{13} \mathrm{C}\left\{{ }^{1} \mathrm{H}\right\} \mathrm{NMR}\left(75 \mathrm{MHz}, \mathrm{CDCl}_{3}\right) \delta 167.4,139.4,134.3,132.1$, 123.7, 114.9, 34.1, 32.1, 29.8, 29.7, 29.4, 29.3, 29.1,27.9, 27.3, 22.8, 14.3; HRMS m/z (ESI) calculated[M] ${ }^{\circ+}=$ 341.2356, measured 341.2355 .

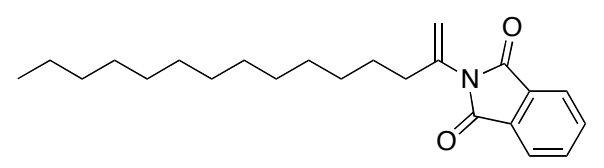

2-(1-Methylene-heptyl)-isoindole-1,3-dione. Column chromatography (hexanes/ether, 4:1) afforded a white solid, $67 \%$ (174 mg). Characterization data agrees with literature report. ${ }^{10}$

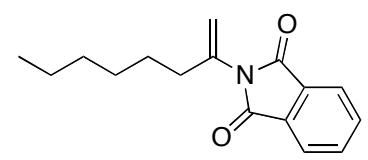

2-Cyclopent-2-enyl-isoindole-1,3-dione. Column chromatography (hexanes/ether, 4:1) afforded a white solid, 83\% $(177 \mathrm{mg})$. Characterization data agrees with literature report. ${ }^{11}$<smiles>O=C1c2ccccc2C(=O)N1C1C=CCC1</smiles>

Cyclopent-2-enyl-sulfamic acid 2,2,2-trichloro-ethyl ester. Column chromatography (hexanes/ether, 4:1) afforded a white solid, 54\% (243 mg). Major Isomer: ${ }^{1} \mathrm{H}$ NMR (300 MHz, $\left.\mathrm{CDCl}_{3}\right) \delta$ 6.05-6.01 (m, 1H), 5.82-5.79 (m, 1H), 4.68-4.57 (m, 2H), 4.64 (s, 2H), 2.54-2.26 (m, 3H), 1.88-1.75 (m. 1H); (75 MHz, $\left.\mathrm{CDCl}_{3}\right) \delta$ 136.5, 130.0, 93.7, 78.4, 61.5, 31.5, 31.2; Minor Isomer (integrations relative to the major isomer): ${ }^{1} \mathrm{H} \mathrm{NMR}\left(300 \mathrm{MHz}, \mathrm{CDCl}_{3}\right) \delta 5.72(\mathrm{~s}$, 0.2), 4.68-4.57 (m, 0.2H), $4.63(\mathrm{~s}, 0.2 \mathrm{H}), 4.30-4.19(\mathrm{~m}, 0.12 \mathrm{H}), 2.79$ (dd, J = 15.3, 7.2 Hz, 0.2H), 2.54-2.26 (m, $0.2 \mathrm{H})$; Major Isomer: ${ }^{13} \mathrm{C}\left\{{ }^{1} \mathrm{H}\right\} \mathrm{NMR}\left(75 \mathrm{MHz} \mathrm{CDCl}_{3}\right) \delta 136.5,129.9,93.9,78.3,61.4,31.4$, 31.1; Minor Isomer: ${ }^{13} \mathrm{C}\left\{{ }^{1} \mathrm{H}\right\} \mathrm{NMR}\left(75 \mathrm{MHz}, \mathrm{CDCl}_{3}\right) \delta 128.7,93.7,78.3,54.7,40.2$; Melting Point 59-60 ${ }^{\circ} \mathrm{C} ; \mathrm{HRMS} \mathrm{m} / \mathrm{z}$ (ESI) calculated $[\mathrm{M}+\mathrm{Na}]^{\bullet+}=315.9345$, measured 315.9332 .<smiles>O=S(=O)(NC1C=CCC1)OCC(Cl)(Cl)Cl</smiles>

2-Cyclooct-2-enyl-isoindole-1,3-dione. Column chromatography (hexanes/ether, 4:1) afforded a white solid, 44\% $(113 \mathrm{mg})$. Characterization data agrees with literature report. ${ }^{10}$

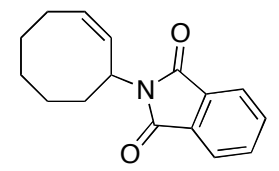


Cyclooct-2-enyl-sulfamic acid 2,2,2-trichloro-ethyl ester. Column chromatography (ether/hexane, 1:4) afforded a white solid, $63 \%$ (211 mg). Allyl Isomer (integrations relative to the homoallyl isomer): ${ }^{1} \mathrm{H} \mathrm{NMR} \mathrm{(500} \mathrm{MHz,}$ $\left.\mathrm{CDCl}_{3}\right) \delta 5.79-5.64(\mathrm{~m}, .6 \mathrm{H}), 5.40(\mathrm{ddd}, \mathrm{J}=6.3,5.1,0.9 \mathrm{~Hz}, .29 \mathrm{H}), 4.82(\mathrm{~d}, \mathrm{~J}=4.5 \mathrm{~Hz}, .30 \mathrm{H}), 4.60(\mathrm{~s}, .63 \mathrm{H}), 4.48$ $4.42(\mathrm{~m}, .30 \mathrm{H}), 2.35-2.27(\mathrm{~m}, 0.6 \mathrm{H}), 2.06-1.98(\mathrm{~m}, .6 \mathrm{H}), 1.72-1.24(\mathrm{~m}, 4.8 \mathrm{H})$; Homoallyl Isomer: ${ }^{1} \mathrm{H}$ NMR (500 $\left.\mathrm{MHz}, \mathrm{CDCl}_{3}\right) \delta 5.83(\mathrm{dt}, \mathrm{J}=6.3,4.5 \mathrm{~Hz}, 1 \mathrm{H}), 6.10(\mathrm{dt}, \mathrm{J}=6.6,4.8 \mathrm{~Hz}, 1 \mathrm{H}), 4.76(\mathrm{~d}, \mathrm{~J}=5.1 \mathrm{~Hz}, 1 \mathrm{H}), 4.61(\mathrm{~d}, \mathrm{~J}=0.9$ $\mathrm{Hz}, 2 \mathrm{H}), 3.75-3.86(\mathrm{~m}, 1 \mathrm{H}), 2.51(\mathrm{ddd}, \mathrm{J}=7.8,5.1,2.4 \mathrm{~Hz}, 1 \mathrm{H}), 2.42(\mathrm{dt}, \mathrm{J}=8.1,4.5 \mathrm{~Hz}, 1 \mathrm{H}), 2.17-2.12(\mathrm{~m}, 4 \mathrm{H})$, 1.92-1.86 (m, 2H), 1.72-1.24 (m, 4H); Allyl Isomer: ${ }^{13} \mathrm{C}\left\{{ }^{1} \mathrm{H}\right\}$ NMR $\left(125 \mathrm{MHz}, \mathrm{CDCl}_{3}\right) \delta$ 131.0, 129.8, 93.8, 78.4, 55.7, 36.1, 26.2, 25.9, 24.5, 23.3; Homoallyl Isomer: ${ }^{13} \mathrm{C}\left\{{ }^{1} \mathrm{H}\right\}$ NMR $\left(125 \mathrm{MHz}, \mathrm{CDCl}_{3}\right) \delta 134.5,125.1,93.9,78.4$, 56.2, 32.6, 31.4, 28.8, 26.3, 22.5; Melting Point $72-75^{\circ} \mathrm{C}$; HRMS m/z (ESI) calculated $[\mathrm{M}+\mathrm{Na}]^{{ }^{+}}=357.9814$, measured 357.9819 .

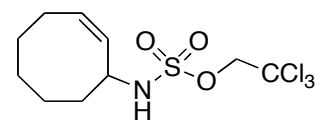

2-(1-Phenyl-vinyl)-isoindole-1,3-dione. Column chromatography (hexanes/ether, 4:1) afforded a white solid, 24\% $(58.5 \mathrm{mg})$. Characterization data agrees with literature report. ${ }^{10}$

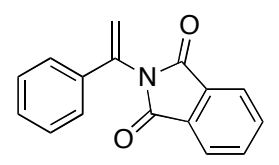

2-(1-Benzyloxymethyl-vinyl)-isoindole-1,3-dione. Column chromatography (ether/hexanes, 1:4) afforded a white solid, 68\% ( $296.2 \mathrm{mg}) .{ }^{1} \mathrm{H}$ NMR (300 MHz, $\left.\mathrm{CDCl}_{3}\right) \delta 7.87$ (dd, J = 5.4, 3.0 Hz, 2H), 7.74 (dd, J = 5.7, 3.0 Hz, 2H), 7.29-7.23 (m, 5H), $5.66(\mathrm{t}, \mathrm{J}=1.2 \mathrm{~Hz}, 1 \mathrm{H}), 5.44(\mathrm{~s}, 1 \mathrm{H}), 4.53(\mathrm{~s}, 2 \mathrm{H}), 4.35(\mathrm{~d}, \mathrm{~J}=1.2 \mathrm{~Hz}, 2 \mathrm{H}) ;{ }^{13} \mathrm{C}\left\{{ }^{1} \mathrm{H}\right\} \mathrm{NMR}(75$ $\left.\mathrm{MHz}, \mathrm{CDCl}_{3}\right) \delta 167.2,137.9,135.6,134.4,132.0,128.5,128.0,127.8,123.8,117.0,72.1,69.1$; Melting Point 60-61 ${ }^{\circ} \mathrm{C} ; \mathrm{HRMS} \mathrm{m} / \mathrm{z}(\mathrm{ESI})$ calculated $[\mathrm{M}+\mathrm{Na}]^{*+}=316.0950$, measured 316.0956 .<smiles>C=C(COCc1ccccc1)N1C(=O)c2ccccc2C1=O</smiles>

2-[1-(2-Benzyloxy-ethyl)-vinyl]-isoindole-1,3-dione. Column chromatography (ether/hexanes, 1:3) afforded a white solid, 63\% (194.3 mg). ). ${ }^{1} \mathrm{H}$ NMR (300 MHz, $\left.\mathrm{CDCl}_{3}\right) \delta 7.82(\mathrm{dd}, \mathrm{J}=5.7,3.3,2 \mathrm{H}), 7.70$ (dd, J = 5.4, 3.0, 2H), 7.20-7.16 (m, 5H), $5.46(\mathrm{~s}, 1 \mathrm{H}), 5.22(\mathrm{~s}, 1 \mathrm{H}), 4.40(\mathrm{~s}, 2 \mathrm{H}), 3.60(\mathrm{t}, \mathrm{J}=6.3 \mathrm{~Hz}, 2 \mathrm{H}), 2.81(\mathrm{td}, \mathrm{J}=6.3,0.9 \mathrm{~Hz}, 2 \mathrm{H})$; ${ }^{13} \mathrm{C}\left\{{ }^{1} \mathrm{H}\right\}$ NMR $\left(75 \mathrm{MHz}, \mathrm{CDCl}_{3}\right) \delta 167.3,138.0,136.6,134.2,131.9,128.3,127.7,127.5,123.6,116.6,73.1,68.1$, 34.1; Melting Point $46-48^{\circ} \mathrm{C}$; HRMS m/z (ESI) calculated $[\mathrm{M}+\mathrm{Na}]^{\circ+}=330.1106$, measured 330.1116.<smiles>C=C(CCOCc1ccccc1)N1C(=O)c2ccccc2C1=O</smiles>

Acetic acid 4-(1,3-dioxo-1,3-dihydro-isoindol-2-yl)-pent-4-enyl ester. Column chromatography (hexanes/ether, 4:1) afforded a white solid, 67\% (181.0 mg). Major Isomer: ${ }^{1} \mathrm{H}$ NMR (300 MHz, $\left.\mathrm{CDCl}_{3}\right) \delta$ 7.90-7.86 (m, 2H), 7.78$7.43(\mathrm{~m}, 2 \mathrm{H}), 5.42(\mathrm{~s}, 1 \mathrm{H}), 5.19(\mathrm{~s}, 1 \mathrm{H}), 4.10(\mathrm{t}, \mathrm{J}=6.6 \mathrm{~Hz}, 2 \mathrm{H}), 2.56(\mathrm{t}, \mathrm{J}=8.1 \mathrm{~Hz}, 2 \mathrm{H}), 2.02(\mathrm{~s}, 3 \mathrm{H}), 1.84-1.75(\mathrm{~m}$, $2 \mathrm{H})$. Minor Isomer (integrations relative to the major isomer): ${ }^{1} \mathrm{H}$ NMR $\left(300 \mathrm{MHz}, \mathrm{CDCl}_{3}\right) \delta 5.57-5.51(\mathrm{~m}, 0.12 \mathrm{H})$, $4.19(\mathrm{t}, \mathrm{J}=6.9 \mathrm{~Hz}, 0.3 \mathrm{H}), 2.08(\mathrm{~s}, 0.4 \mathrm{H})$; Major Isomer: ${ }^{13} \mathrm{C}\left\{{ }^{1} \mathrm{H}\right\} \mathrm{NMR}\left(75 \mathrm{MHz}, \mathrm{CDCl}_{3}\right) \delta$ 171.2, 67.4, 134.55, 134.51, 123.8, 115.8, 63.6, 30.6, 26.1, 21.1. Minor Isomer: ${ }^{13} \mathrm{C}\left\{{ }^{1} \mathrm{H}\right\}$ NMR $\left(75 \mathrm{MHz}, \mathrm{CDCl}_{3}\right) \delta$ 168.0, 167.5, 138.0, 134.3, 131.9, 127.3, 63.1, 29.9, 27.6, 16.1; Melting Point $64-68^{\circ} \mathrm{C}$; HRMS m/z (ESI) calculated $[\mathrm{M}+\mathrm{Na}]^{0^{++}}=$ 296.0899, measured 296.0909.

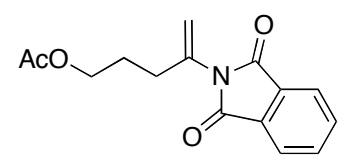


2-(1-Oxiranylmethoxymethyl-vinyl)-isoindole-1,3-dione. Column chromatography (ethyl acetate/hexanes, 1:2) afforded a yellow oil, 63\% (235.4 mg). ${ }^{1} \mathrm{H}$ NMR $\left(300 \mathrm{MHz}, \mathrm{CDCl}_{3}\right) \delta 7.89(\mathrm{dd}, \mathrm{J}=5.7,3 \mathrm{~Hz}, 2 \mathrm{H}), 7.74(\mathrm{dd}, \mathrm{J}=5.4$, $3 \mathrm{~Hz}, 2 \mathrm{H}), 5.66(\mathrm{~s}, 1 \mathrm{H}), 5.42(\mathrm{~s}, 1 \mathrm{H}), 4.38(\mathrm{q}, \mathrm{J}=12.9 \mathrm{~Hz}, 2 \mathrm{H}), 3.77(\mathrm{dd}, \mathrm{J}=11.4,3 \mathrm{~Hz}, 1 \mathrm{H}), 3.42(\mathrm{dd}, \mathrm{J}=11.4,6$ $\mathrm{Hz}, 1 \mathrm{H}), 3.12-3.06(\mathrm{~m}, 1 \mathrm{H}), 2.73(\mathrm{dd}, \mathrm{J}=5.1,4.5 \mathrm{~Hz}, 1 \mathrm{H}), 2.56(\mathrm{dd}, \mathrm{J}=5.1,4.5 \mathrm{~Hz}, 1 \mathrm{H}) ;{ }^{13} \mathrm{C}\left\{{ }^{1} \mathrm{H}\right\} \mathrm{NMR}(75 \mathrm{MHz}$, $\left.\mathrm{CDCl}_{3}\right) \delta 167.3,135.3,134.5,132.8,117.4,70.8,70.3,50.8,44.3 ; \mathrm{HRMS} \mathrm{m} / \mathrm{z}(\mathrm{ESI})$ calculated $[\mathrm{M}+\mathrm{Na}]^{*+}=$ 282.0742, measured 282.0743.<smiles>C=C(COCC1CO1)N1C(=O)c2ccccc2C1=O</smiles>

2-[1-(2-Oxiranyl-ethyl)-vinyl]-isoindole-1,3-dione. Column chromatography (hexanes/EtOAc, 3:1) afforded a colorless oil, 76\% (179.8 mg). Major Isomer ${ }^{1} \mathrm{H}$ NMR $\left(300 \mathrm{MHz}, \mathrm{CDCl}_{3}\right) \delta 7.89$ (dd, J = 5.4, $\left.3.0 \mathrm{~Hz}, 2 \mathrm{H}\right), 7.76$ (dd, $\mathrm{J}=5.4,3.0 \mathrm{~Hz}, 2 \mathrm{H}), 5.54(\mathrm{t}, \mathrm{J}=1.0 \mathrm{~Hz}, 1 \mathrm{H}), 5.21(\mathrm{~s}, 1 \mathrm{H}), 3.01-2.95(\mathrm{~m}, 1 \mathrm{H}), 2.75(\mathrm{dd}, \mathrm{J}=4.5,3.9 \mathrm{~Hz}, 1 \mathrm{H}), 2.66$ $(\mathrm{qd}, \mathrm{J}=6.0,0.6 \mathrm{~Hz}, 2 \mathrm{H}), 2.94(\mathrm{dd}, \mathrm{J}=4.8,2.7 \mathrm{~Hz}, 1 \mathrm{H}), 1.80-1.60(\mathrm{~m}, 2 \mathrm{H})$; Major Isomer ${ }^{13} \mathrm{C}\left\{{ }^{1} \mathrm{H}\right\} \mathrm{NMR}(75 \mathrm{MHz}$, $\left.\mathrm{CDCl}_{3}\right) \delta 167.3,138.0,134.4,131.9,123.8,115.8,51.5,47.3,30.5,30.0 ;$ HRMS m/z $(\mathrm{ESI})$ calculated $[\mathrm{M}+\mathrm{Na}]^{*+}=$ 266.0793, measured 266.0786.<smiles>C=C(CCC1CO1)N1C(=O)c2ccccc2C1=O</smiles>

2-[1-(2-Hydroxy-ethyl)-vinyl]-isoindole-1,3-dione. Column chromatography (hexanes/EtOAc, 1:1) afforded a white solid, $17 \%$ (36.8 mg). Major Isomer: ${ }^{1} \mathrm{H}$ NMR (300 MHz, $\left.\mathrm{CDCl}_{3}\right) \delta$ 7.92-7.85 (m, 2H), (7.80-7.73 (m, 2H), $5.58(\mathrm{~s}, 1 \mathrm{H}), 5.30(\mathrm{~s}, 1 \mathrm{H}), 3.67(\mathrm{q}, \mathrm{J}=4.5 \mathrm{~Hz}, 2 \mathrm{H}), 2.68(\mathrm{td}, \mathrm{J}=6.0,0.2 \mathrm{~Hz}, 2 \mathrm{H}), 2.34($ broad s, 1H); Minor Isomer (integrations to relative to the major isomer): ${ }^{1} \mathrm{H}$ NMR ( $\left.300 \mathrm{MHz}, \mathrm{CDCl}_{3}\right) \delta 5.78(\mathrm{tq}, \mathrm{J}=6.9,1.2 \mathrm{~Hz}, 0.18 \mathrm{H}) 4.37$ (d, $\mathrm{J}=6.3 \mathrm{~Hz}, 0.38 \mathrm{H}), 2.07$ (broad s, 0.18H), $2.05(\mathrm{~s}, 0.57 \mathrm{H})$; Major Isomer: ${ }^{13} \mathrm{C}\left\{{ }^{1} \mathrm{H}\right\} \mathrm{NMR}\left(75 \mathrm{MHz}, \mathrm{CDCl}_{3}\right) \delta 167.8$, 135.8, 134.6, 131.9, 123.9, 119.8, 59.4, 38.1; HRMS m/z (ESI) calculated $[\mathrm{M}]^{{ }^{+}}=217.0739$, measured 217.0737.<smiles>C=C(CCO)N1C(=O)c2ccccc2C1=O</smiles>

2-\{1-[3-(Triethyl-silanyloxy)-propyl]-vinyl\}-isoindole-1,3-dione. Column chromatography (hexanes/ether, 4:1) afforded a colorless oil, 70\% (240.2 mg). Major Isomer: ${ }^{1} \mathrm{H}$ NMR $\left(300 \mathrm{MHz}, \mathrm{CDCl}_{3}\right) \delta$ 7.89-7.85 (m, 2H), 7.77-7.72 $(\mathrm{m}, 2 \mathrm{H}), 5.41(\mathrm{t}, \mathrm{J}=1.2 \mathrm{~Hz}, 1 \mathrm{H}), 5.16(\mathrm{~s}, 1 \mathrm{H}), 3.63(\mathrm{t}, \mathrm{J}=6.6 \mathrm{~Hz}, 2 \mathrm{H}), 2.55(\mathrm{t}, \mathrm{J}=7.8 \mathrm{~Hz}, 2 \mathrm{H}), 1.72-1.62(\mathrm{~m}, 2 \mathrm{H})$, 0.929 (t, J $=8.1 \mathrm{~Hz}, 9 \mathrm{H}), 0.55(\mathrm{q}, \mathrm{J}=8.1 \mathrm{~Hz}, 6 \mathrm{H})$; Major Isomer: ${ }^{13} \mathrm{C}\left\{{ }^{1} \mathrm{H}\right\} \mathrm{NMR}\left(75 \mathrm{MHz}, \mathrm{CDCl}_{3}\right) \delta 167.3,138.8$, $134.3,132.0,123.6,115.2,61.8,30.4,30.1,6.9,4.5 ; \mathrm{HRMS} \mathrm{m} / \mathrm{z}(\mathrm{ESI})$ calculated $[\mathrm{M}+\mathrm{Na}]^{\circ+}=368.1658$, measured 368.1653 .<smiles>C=C(CCCOC(F)(F)F)N1C(=O)c2ccccc2C1=O</smiles> 
2-\{1-[3-(tert-Butyl-dimethyl-silanyloxy)-propyl]-vinyl\}-isoindole-1,3-dione. Column chromatography (hexanes/ether, 4:1) afforded a colorless oil, 83\% (303.6 mg). Major Isomer: ${ }^{1} \mathrm{H}$ NMR (300 MHz, $\left.\mathrm{CDCl}_{3}\right) \delta 7.88$ $7.82(\mathrm{~m}, 2 \mathrm{H}), 7.75-7.68(\mathrm{~m}, 2 \mathrm{H}), 5.36(\mathrm{t}, \mathrm{J}=1.2 \mathrm{~Hz}, 1 \mathrm{H}), 5.13(\mathrm{~s}, 1 \mathrm{H}), 3.60(\mathrm{t}, \mathrm{J}=6.3 \mathrm{~Hz}, 2 \mathrm{H}), 2.52(\mathrm{t}, \mathrm{J}=7.5 \mathrm{~Hz}$, $2 \mathrm{H}), 1.67-1.58(\mathrm{~m}, 2 \mathrm{H}), 0.84(\mathrm{~s}, 9 \mathrm{H}), 0.00(\mathrm{~s}, 6 \mathrm{H})$; Minor Isomer (integrations relative to the major isomer): ${ }^{1} \mathrm{H}$ NMR $\left(300 \mathrm{MHz}, \mathrm{CDCl}_{3}\right) \delta 5.53(\mathrm{tq}, \mathrm{J}=7.5,1.2 \mathrm{~Hz}, 0.13 \mathrm{H}), 3.70(\mathrm{t}, \mathrm{J}=6.6 \mathrm{~Hz}, 0.28 \mathrm{H}), 2.44(\mathrm{qd}, \mathrm{J}=7.5,0.9 \mathrm{~Hz}$, $0.39 \mathrm{H}), 1.97(\mathrm{~d}, \mathrm{~J}=1.2 \mathrm{~Hz}, 0.38 \mathrm{H}), 0.87(\mathrm{~s}, 1.09 \mathrm{H}), 0.05(\mathrm{~s}, 0.68 \mathrm{H})$; Major Isomer: ${ }^{13} \mathrm{C}\left\{{ }^{1} \mathrm{H}\right\} \mathrm{NMR}(75 \mathrm{MHz}$, $\left.\mathrm{CDCl}_{3}\right) \delta 167.5,138.8,134.3,132.0,123.7,115.3,62.1,30.4,30.1,26.1,18.4,-5.1 ; \mathrm{HRMS} \mathrm{m} / \mathrm{z}$ (ESI) calculated $[\mathrm{M}+\mathrm{Na}]^{\circ+}=368.1658$, measured 368.1647.<smiles>C=C(CCCOCCCCCCCC)N1C(=O)c2ccccc2C1=O</smiles>

2-\{1-[2-(tert-Butyl-dimethyl-silanyoxy)-ethyl]-vinyl\}-isoindole-1.3-dione. Column chromatography (ether/hexanes, 6:1) afforded a white solid, $77 \%(255.3 \mathrm{mg}) .{ }^{1} \mathrm{H}$ NMR $\left(300 \mathrm{MHz}, \mathrm{CDCl}_{3}\right) \delta 7.94(\mathrm{dd}, \mathrm{J}=5.4,3 \mathrm{~Hz}$, 2H), $7.81(\mathrm{dd}, \mathrm{J}=5.7,3 \mathrm{~Hz}, 2 \mathrm{H}), 5.49(\mathrm{~s}, 1 \mathrm{H}), 5.28(\mathrm{~s}, 1 \mathrm{H}), 3.83(\mathrm{t}, \mathrm{J}=6.3 \mathrm{~Hz}, 2 \mathrm{H}), 2.78(\mathrm{t}, \mathrm{J}=6.3 \mathrm{~Hz}, 2 \mathrm{H}), 0.84(\mathrm{~s}$, 9H), $0.01(\mathrm{~s}, 6 \mathrm{H}) ;{ }^{13} \mathrm{C}\left\{{ }^{1} \mathrm{H}\right\}$ NMR $\left(75 \mathrm{MHz}, \mathrm{CDCl}_{3}\right) \delta 167.3,136.9,134.2,132.2,123.6,116.4,61.7,37.2,25.9,18.3$, $-5.4 ; \mathrm{HRMS} \mathrm{m} / \mathrm{z}(\mathrm{ESI})$ calculated $[\mathrm{M}+\mathrm{Na}]^{*+}=354.1501$, measured 354.1490 .<smiles>C=C(CCOC)N1C(=O)c2ccccc2C1=O</smiles>

2-[1-(tert-Butyl-dimethyl-silanyloxy)-vinyl]-isoindole-1,3-dione. Column chromatography (hexanes/ether, 4:1) afforded a white solid, $76 \%(245.0 \mathrm{mg}) .{ }^{1} \mathrm{H}$ NMR $\left(300 \mathrm{MHz}, \mathrm{CDCl}_{3}\right) \delta 7.83(\mathrm{dd}, \mathrm{J}=5.4,3.3 \mathrm{~Hz}, 2 \mathrm{H}), 7.69(\mathrm{dd}, \mathrm{J}=$ $5.4,3.3 \mathrm{~Hz}, 2 \mathrm{H}), 5.62(\mathrm{t}, \mathrm{J}=1.5 \mathrm{~Hz}, 1 \mathrm{H}), 5.25(\mathrm{t}, \mathrm{J}=1.5 \mathrm{~Hz}, 1 \mathrm{H}), 4.34(\mathrm{t}, \mathrm{J}=1.5 \mathrm{~Hz}, 2 \mathrm{H}), 0.78(\mathrm{~s}, 9 \mathrm{H}), 0.00(\mathrm{~s}, 6 \mathrm{H})$; ${ }^{13} \mathrm{C}\left\{{ }^{1} \mathrm{H}\right\}$ NMR (75 MHz, $\left.\mathrm{CDCl}_{3}\right) \delta 167.3,138.3,134.4,132.1,123.7,114.3,62.3,25.9,18.3,-5.2$; Melting Point 80$82{ }^{\circ} \mathrm{C}$; HRMS m/z (ESI) calculated $[\mathrm{M}+\mathrm{Na}]^{\circ+}=340.1345$, measured 340.1350.

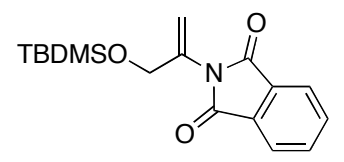

\section{References:}

${ }^{1}$ Cleary, P. A.; Woerpel, K. A. Org. Lett. 2005, 7, 5531-5533.

${ }^{2}$ Raheem, I. T.; Goodman, S. N.; Jacobsen, E. N. J. Am. Chem. Soc. 2004, 126, $706-707$.

${ }^{3}$ Storer, R. I.; Takemoto, T.; Jackson, P. S.; Brown, D. S.; Baxendale, I. R.; Ley, S. V. Chem. Eur. J. 2004, 10, 2529-2547.

${ }^{4}$ Waser, J.; Nambu, H.; Carreira, E. M. J. Am. Chem. Soc. 2005, 127, 8294-8295.

${ }^{5}$ Peters, M. A.; Belu, A. M.; Linton, R. W.; Dupray, L.; Meyer, T. J.; DeSimone, J. M. J. Am. Chem. Soc. 1995, $117,3380-3388$.

${ }^{6}$ Coates, R. M.; Senter, P. D.; Baker, W. R. J. Org. Chem. 1982, 47, 3597-3607.

${ }^{7}$ Velasco-Trooijos, T.; Murphy, P. V. Tetrahedron: Asymmetry 2005, 16, 261-272/

${ }^{8}$ Nishimura, T.; Kakiuchi, N.; Onoue, T.; Ohe, K.; Uemura, S. J. Chem. Soc., Perkin Trans. 1 2000, 1915-1918.

${ }^{9}$ Tokuyasu, T.; Kunikawa, S.; McCullough, K. J.; Masuyama, A.; Nojima, M. J. Org. Chem. 2005, 70, 251-260.

${ }^{10}$ Brice, J. L,; Harang, J. E.; Timokhin, V. I.; Anastasi, N. R.; Stahl, S. S. J. Am. Chem. Soc. 2006, 127, 2868-2869.

${ }^{11}$ Mordini, A.; Russo, F.; Valacchi, M.; Zani, L.; Degl'Innocenti, A.; Reginato, G.; Tetrahedron 2002, 58, 71537163. 

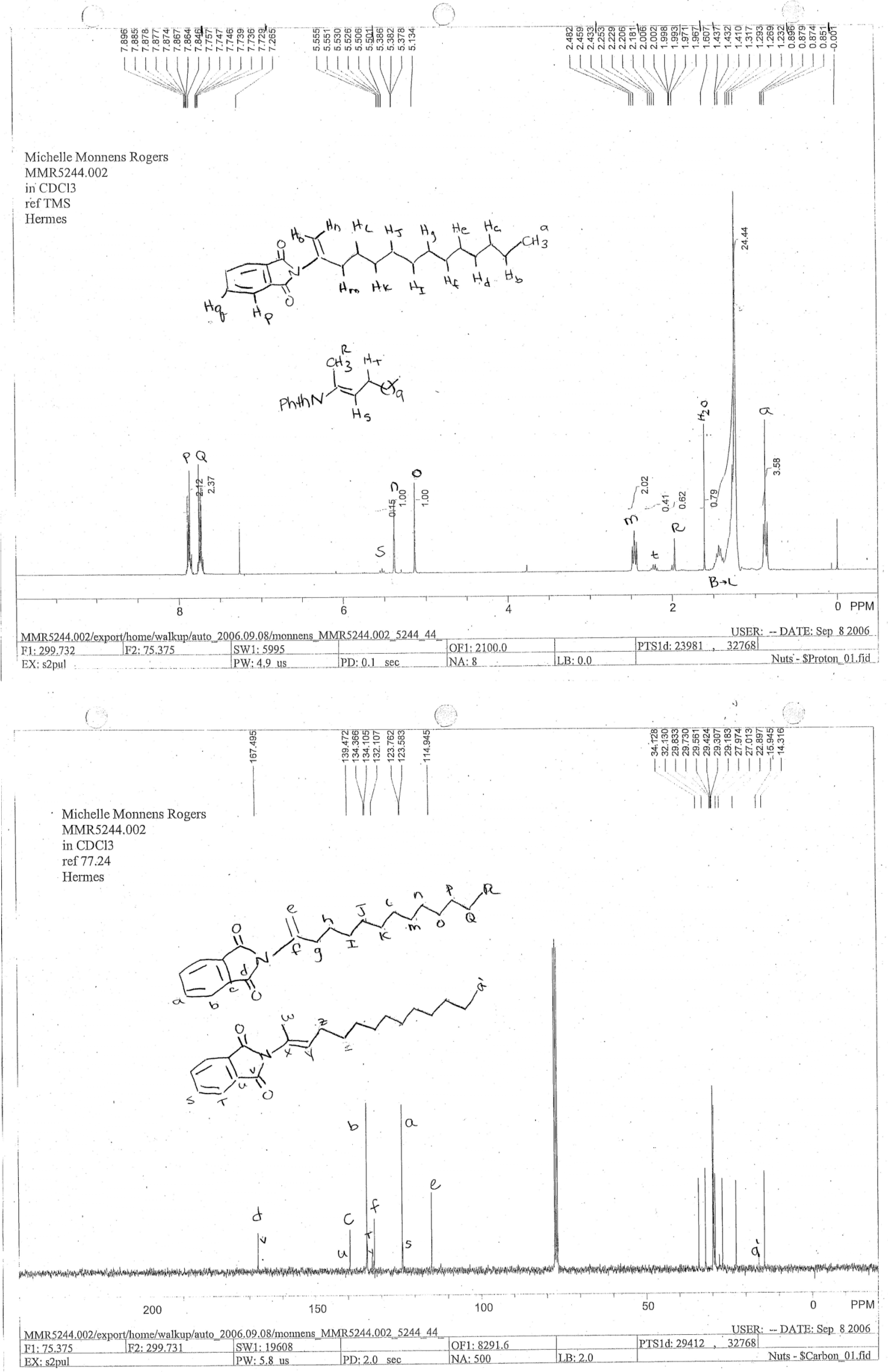

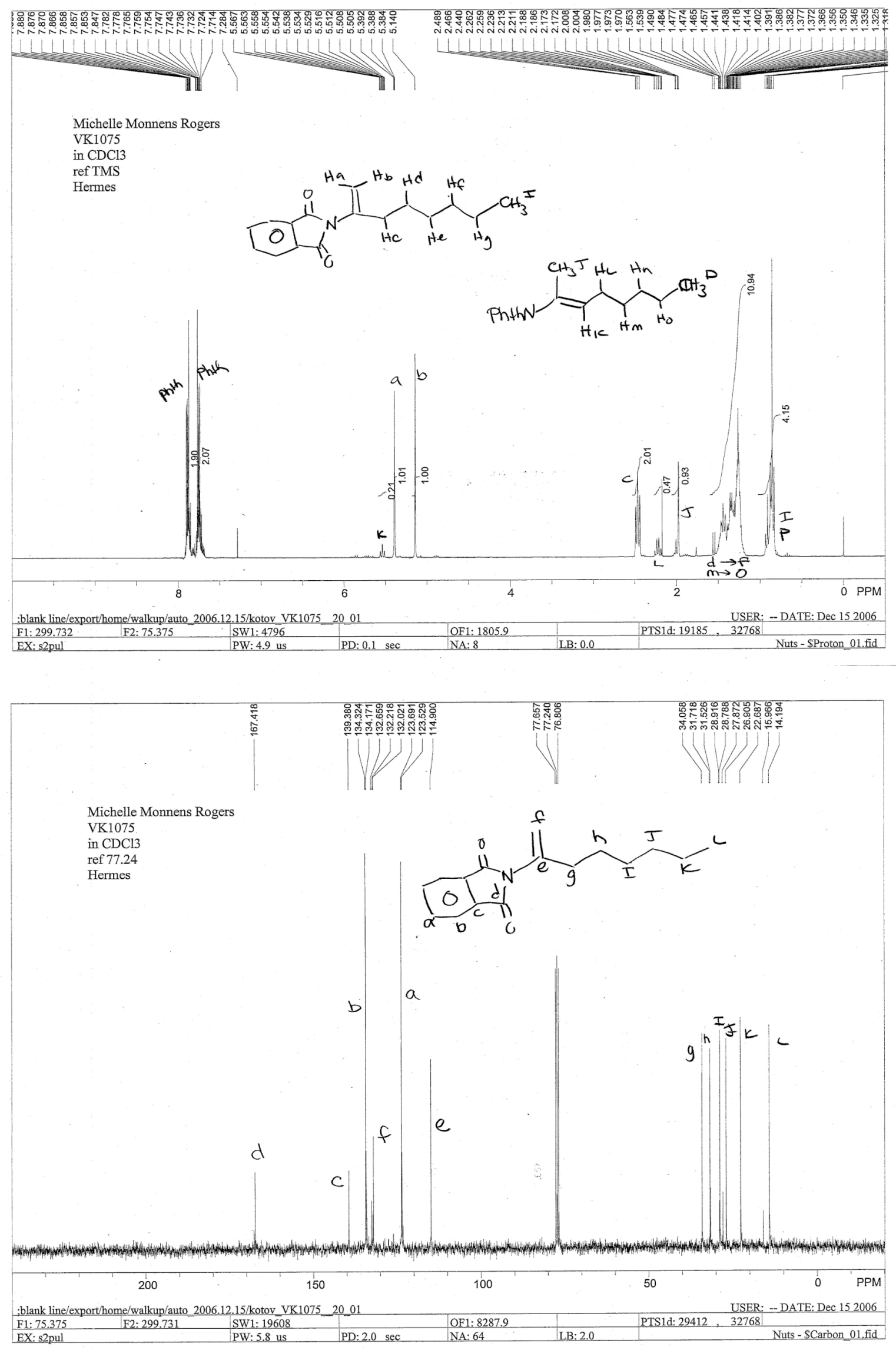

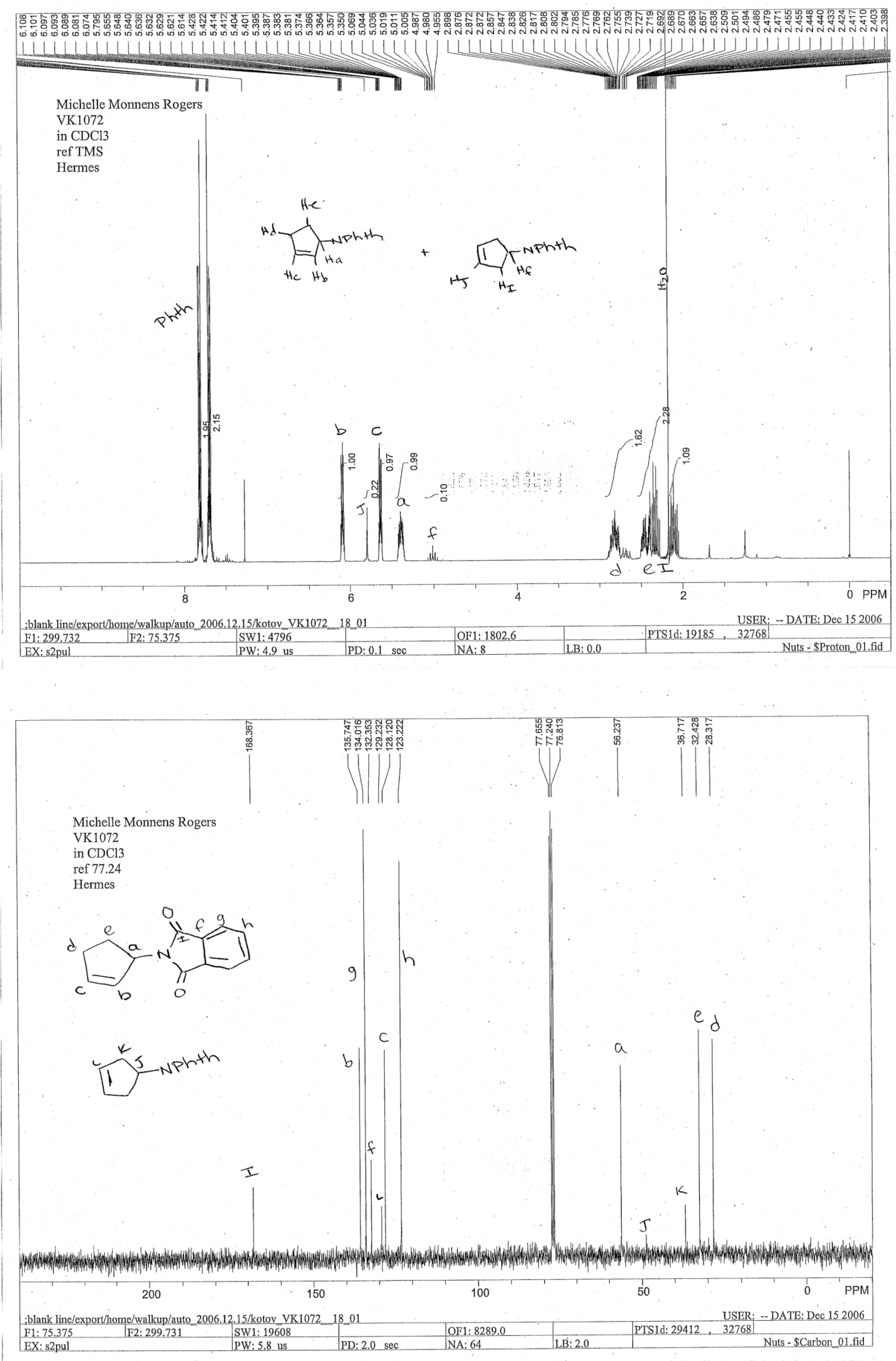

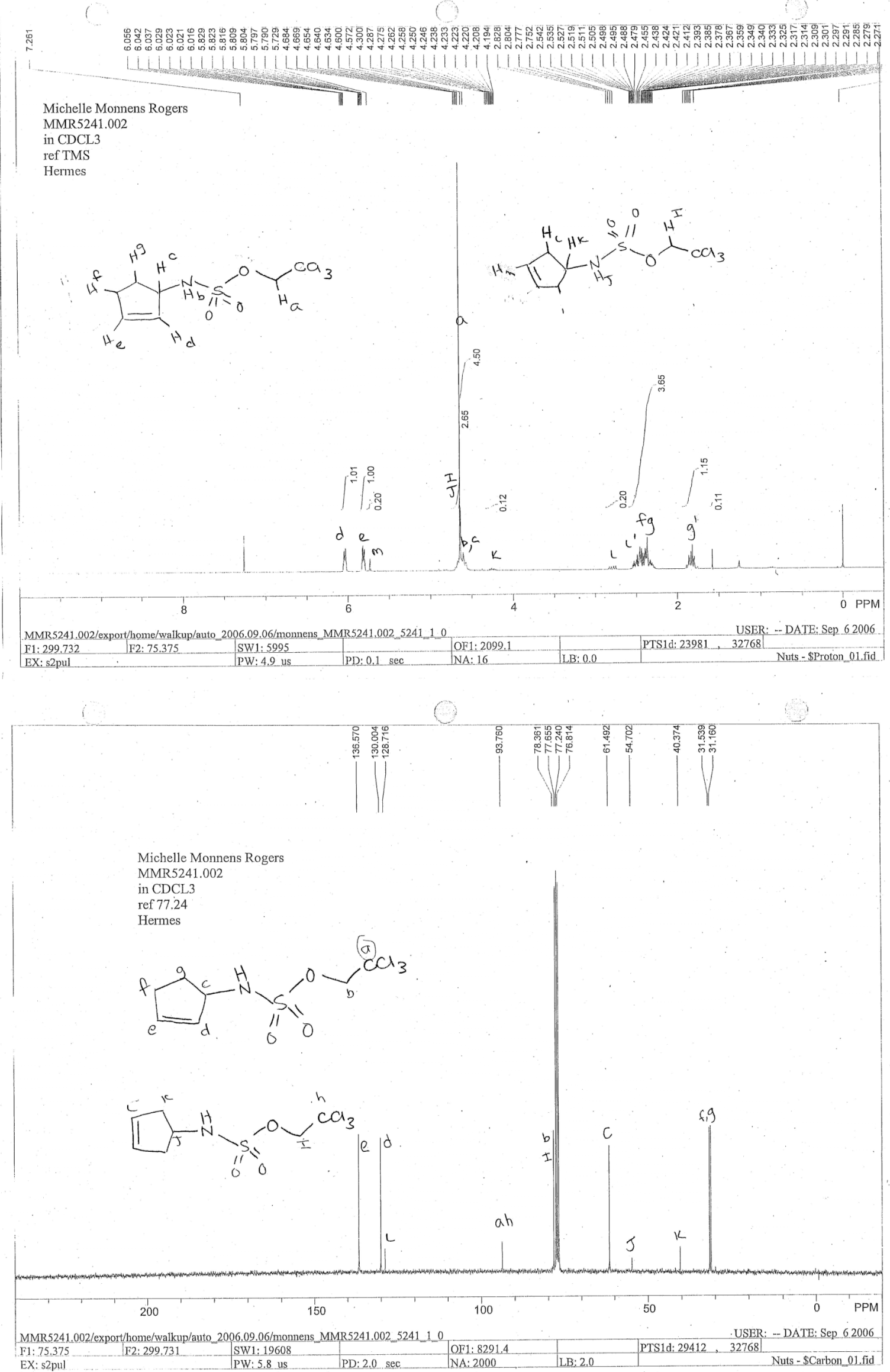

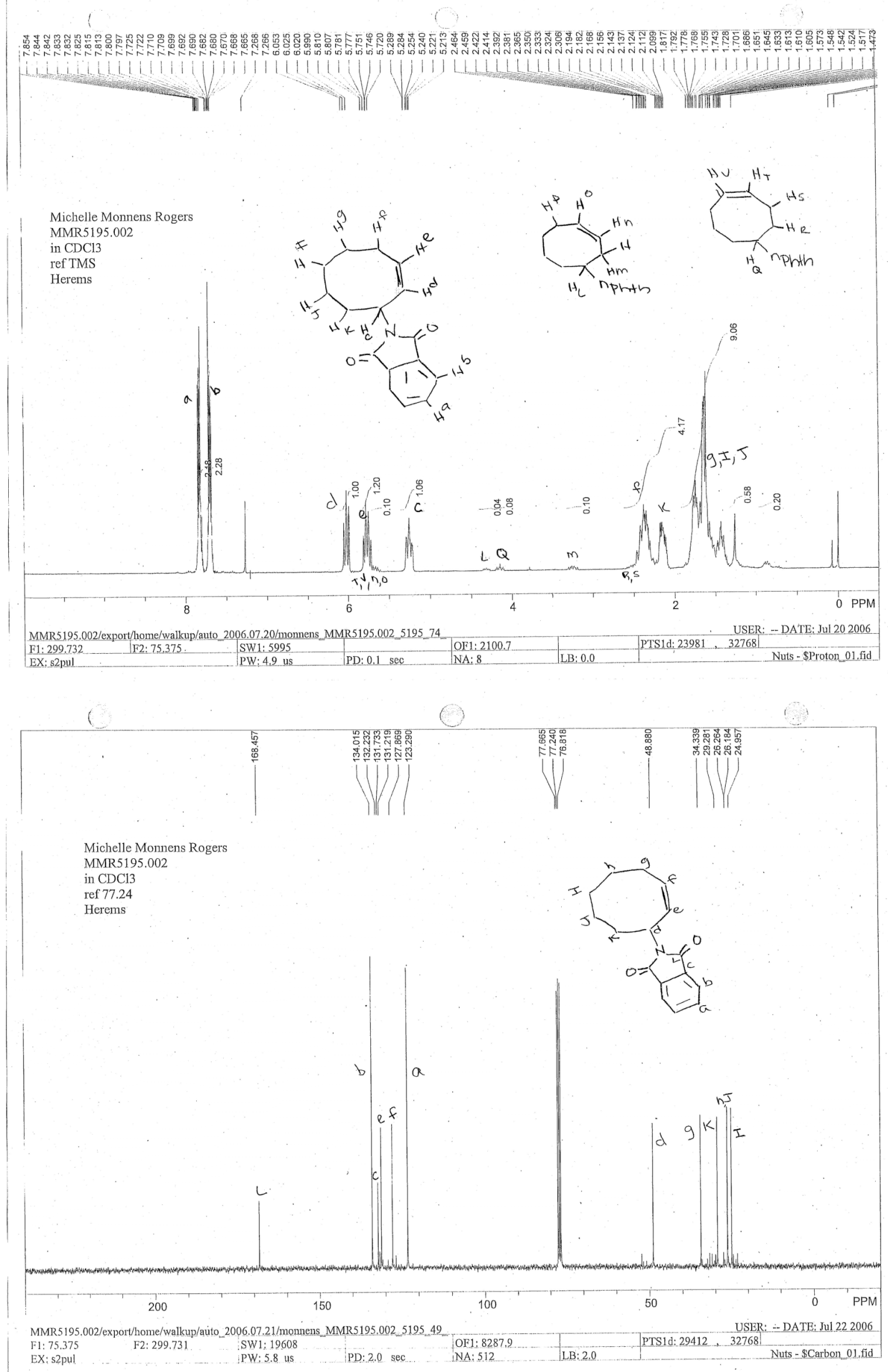

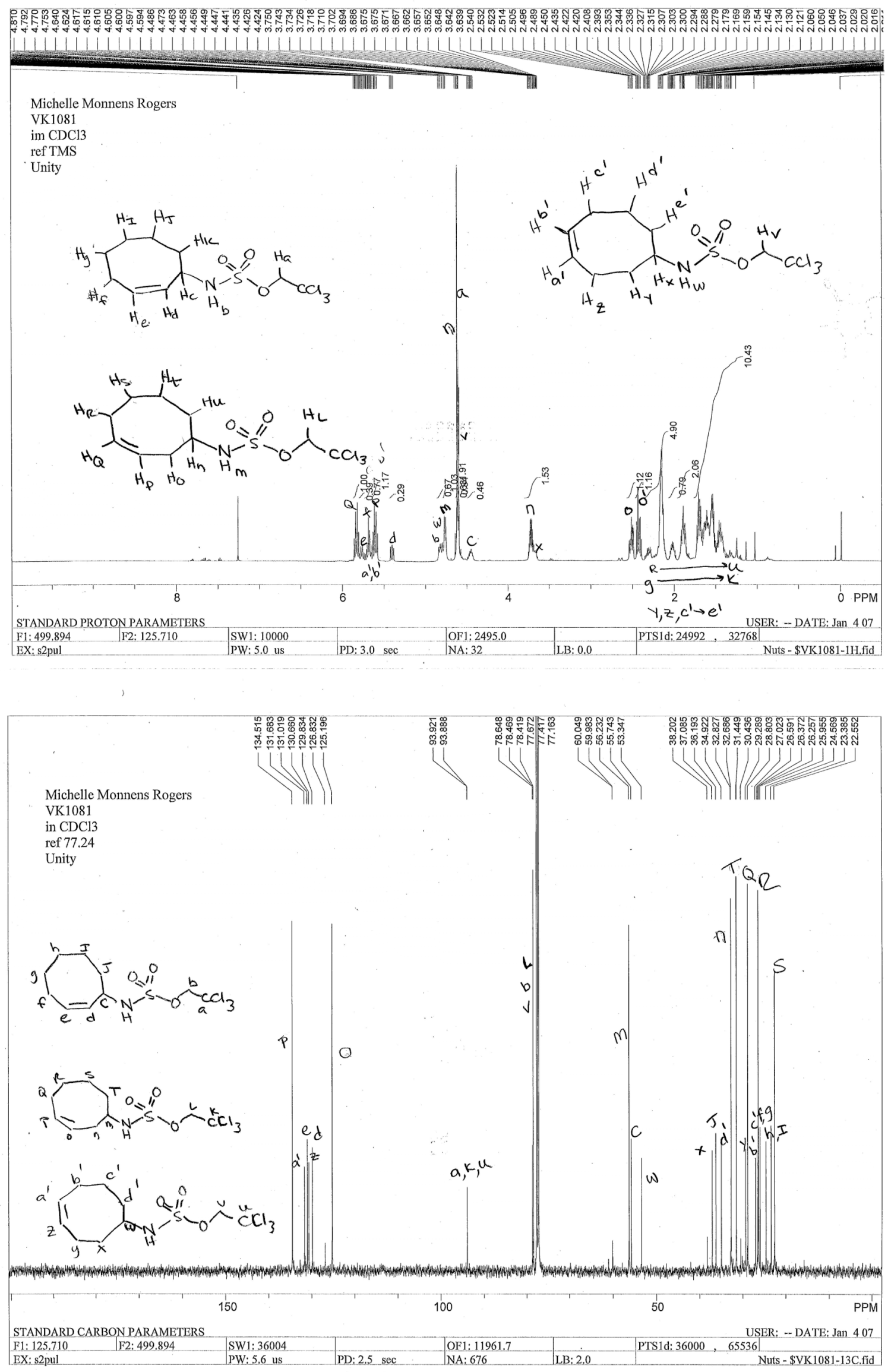

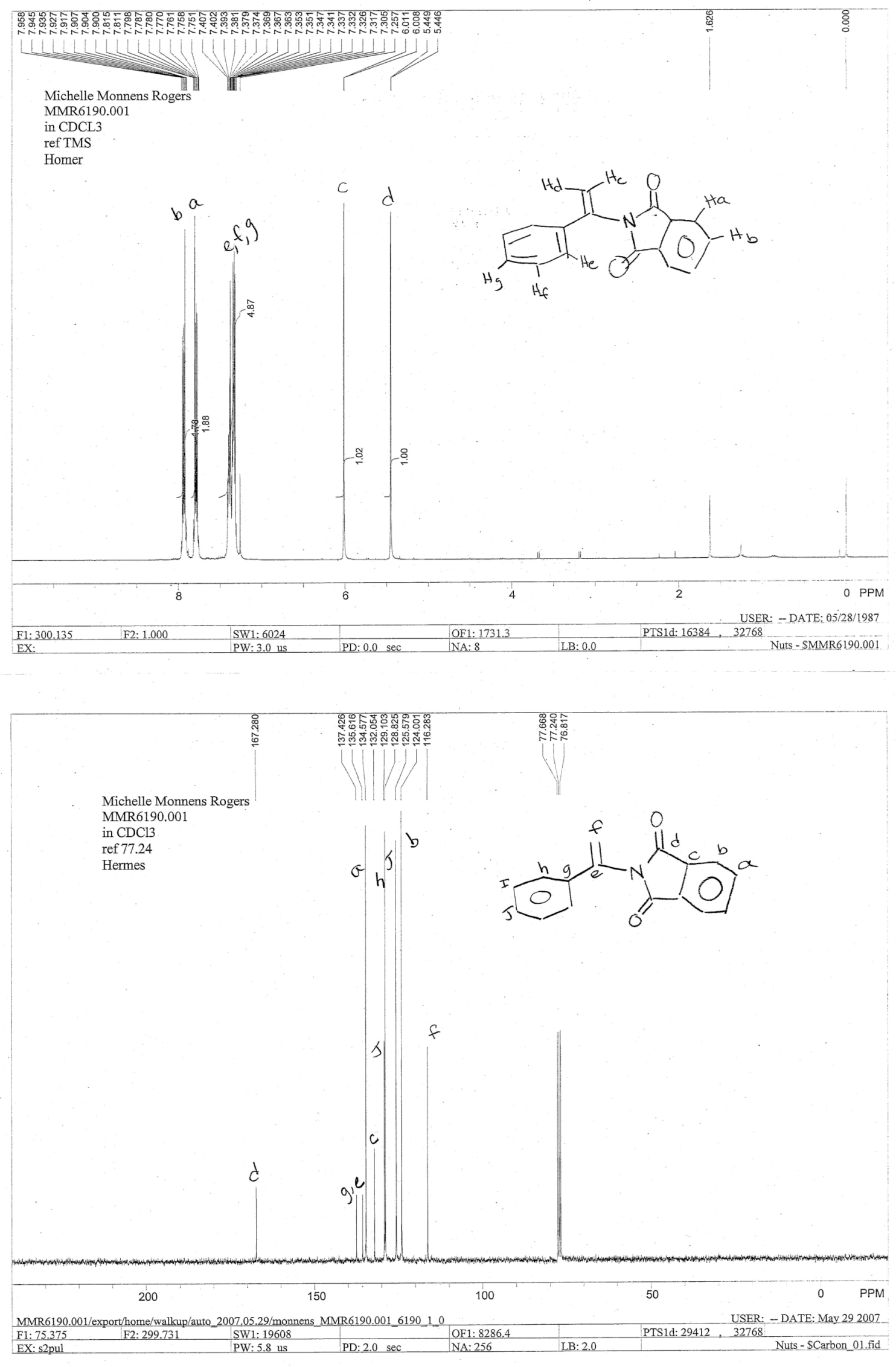

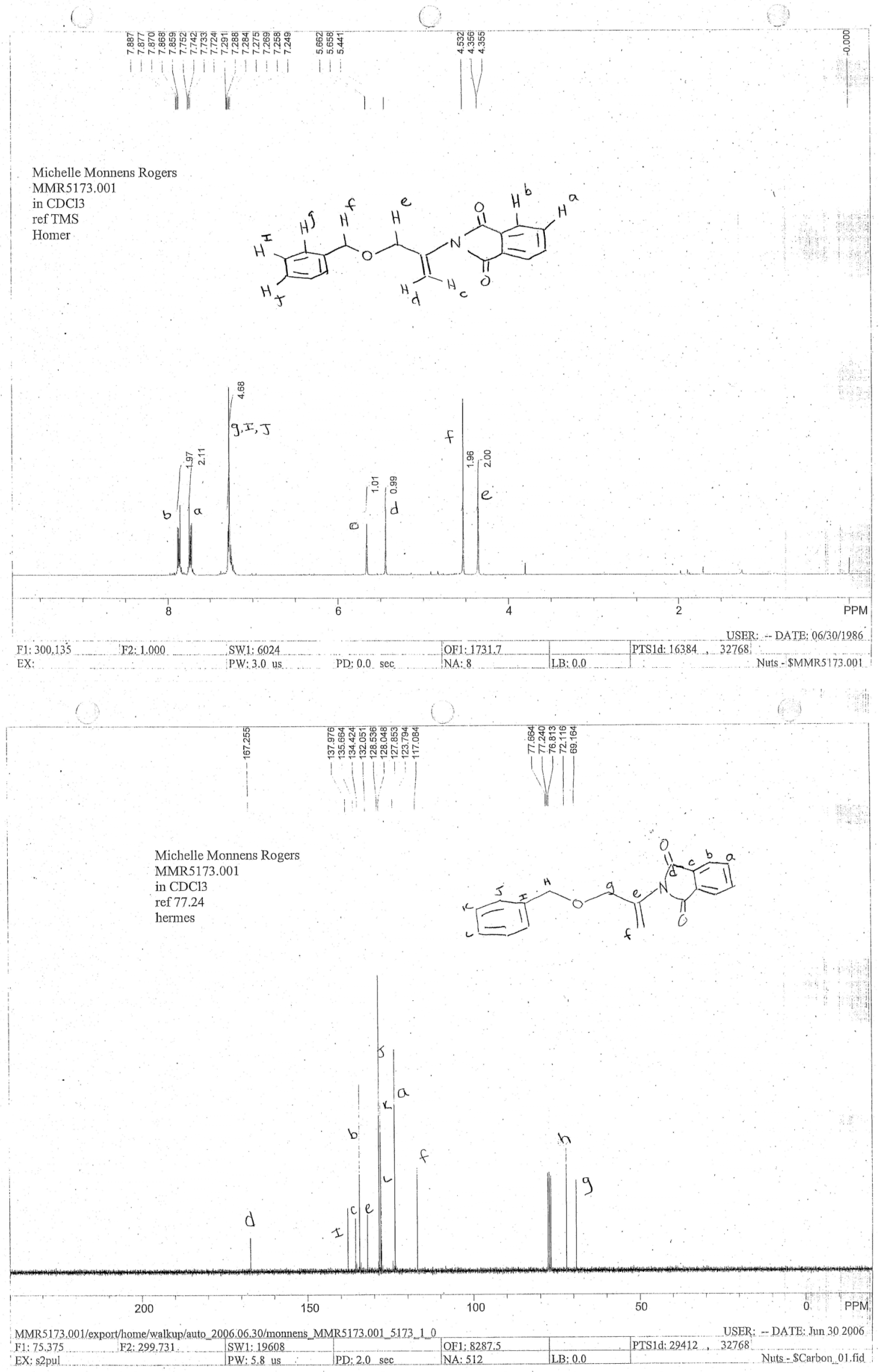

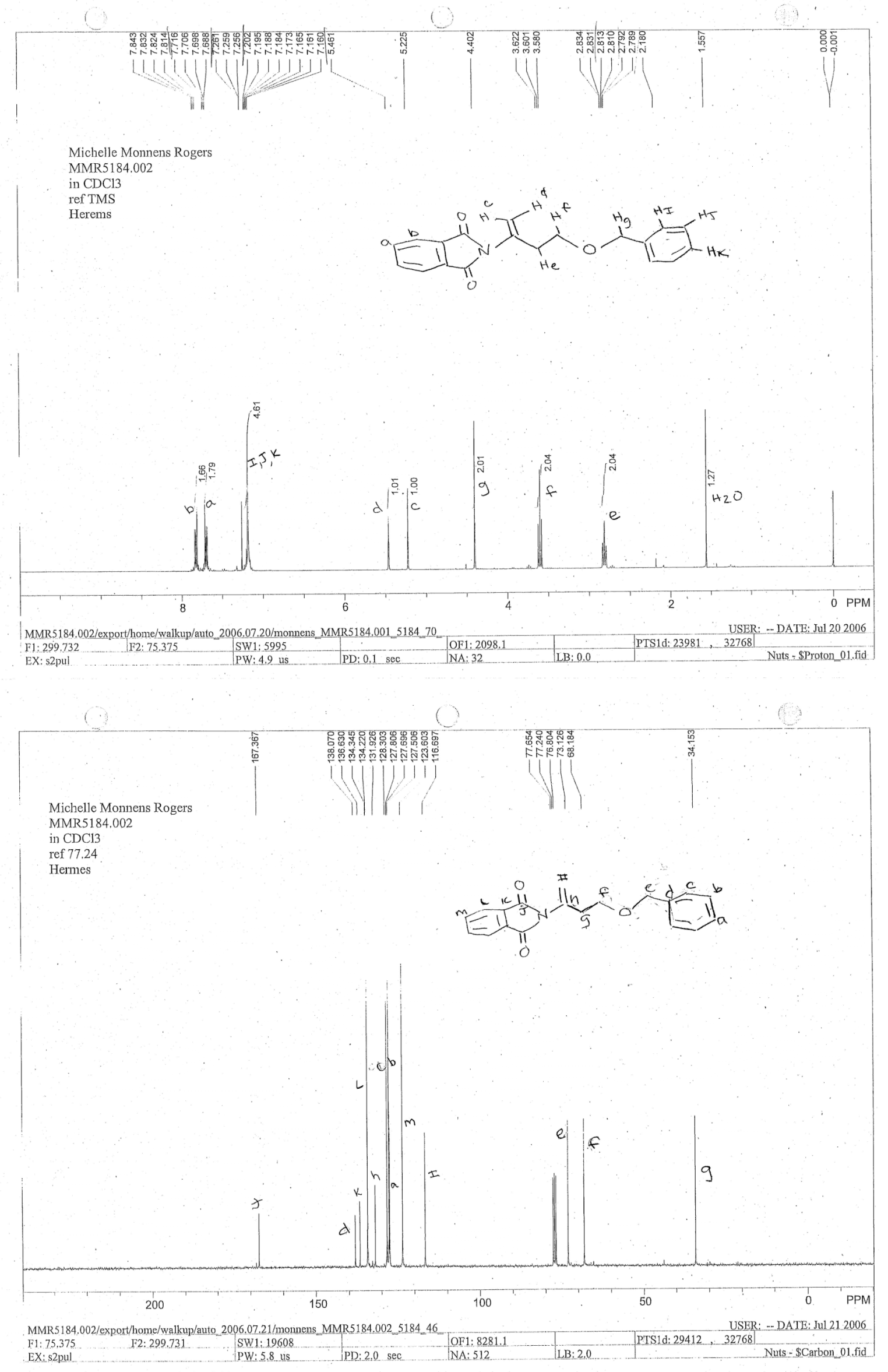

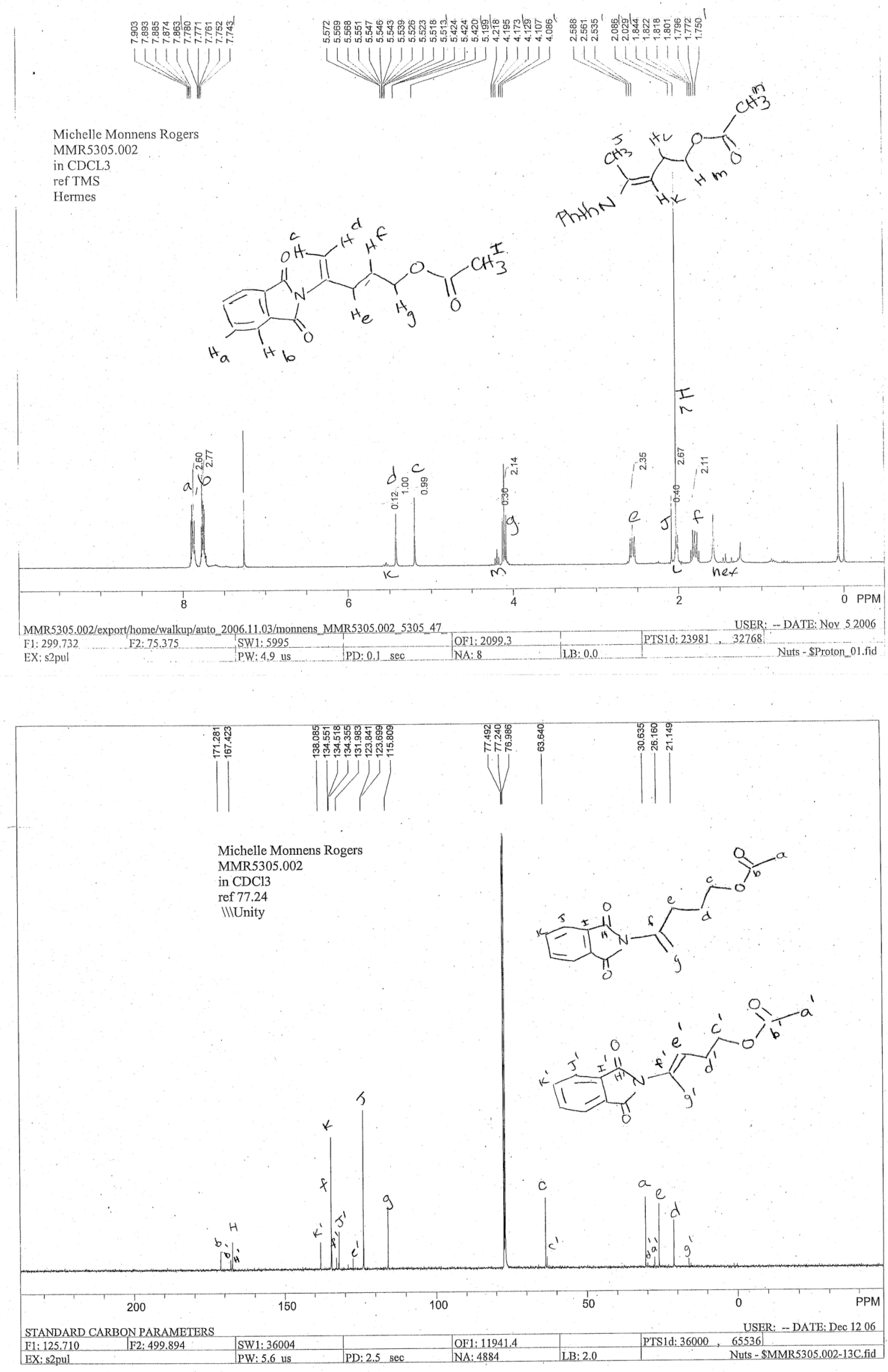

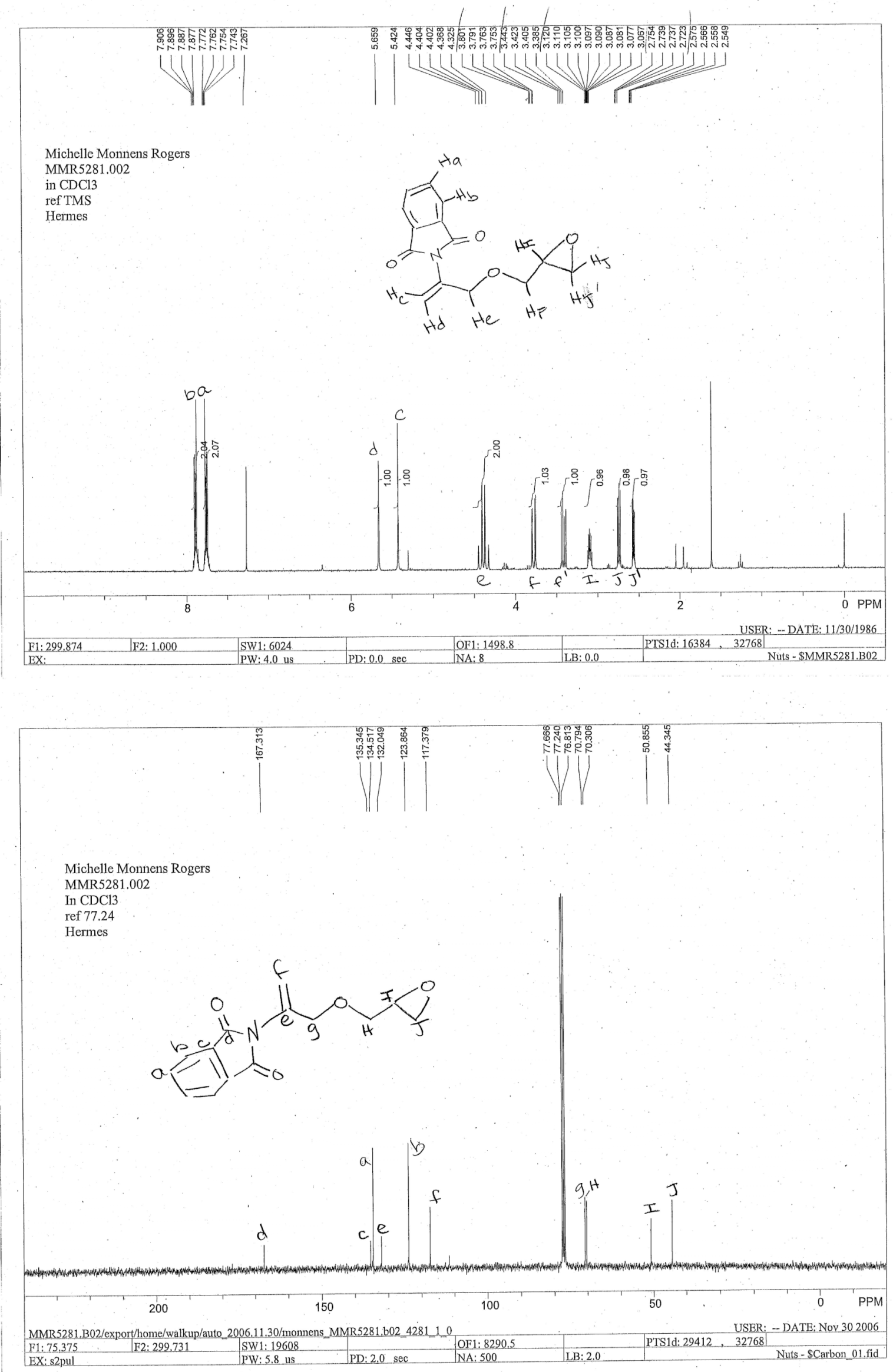
Michelle Monnens Rogers

MMR5306,002

in CDCL3

ref TMS

Athena

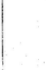

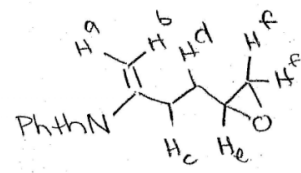

$\mathrm{H}_{c} \mathrm{He}_{e}$

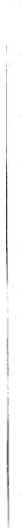
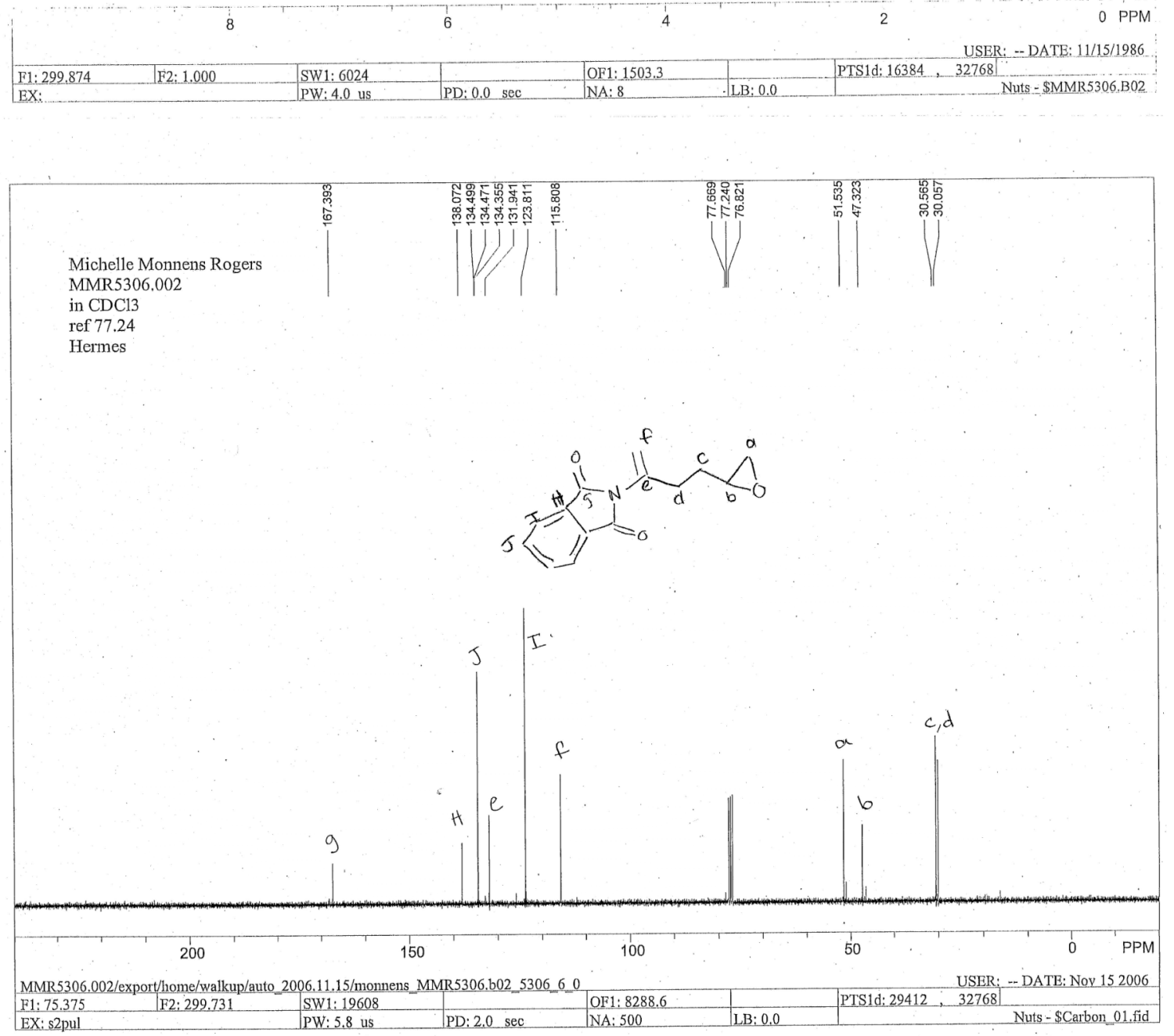

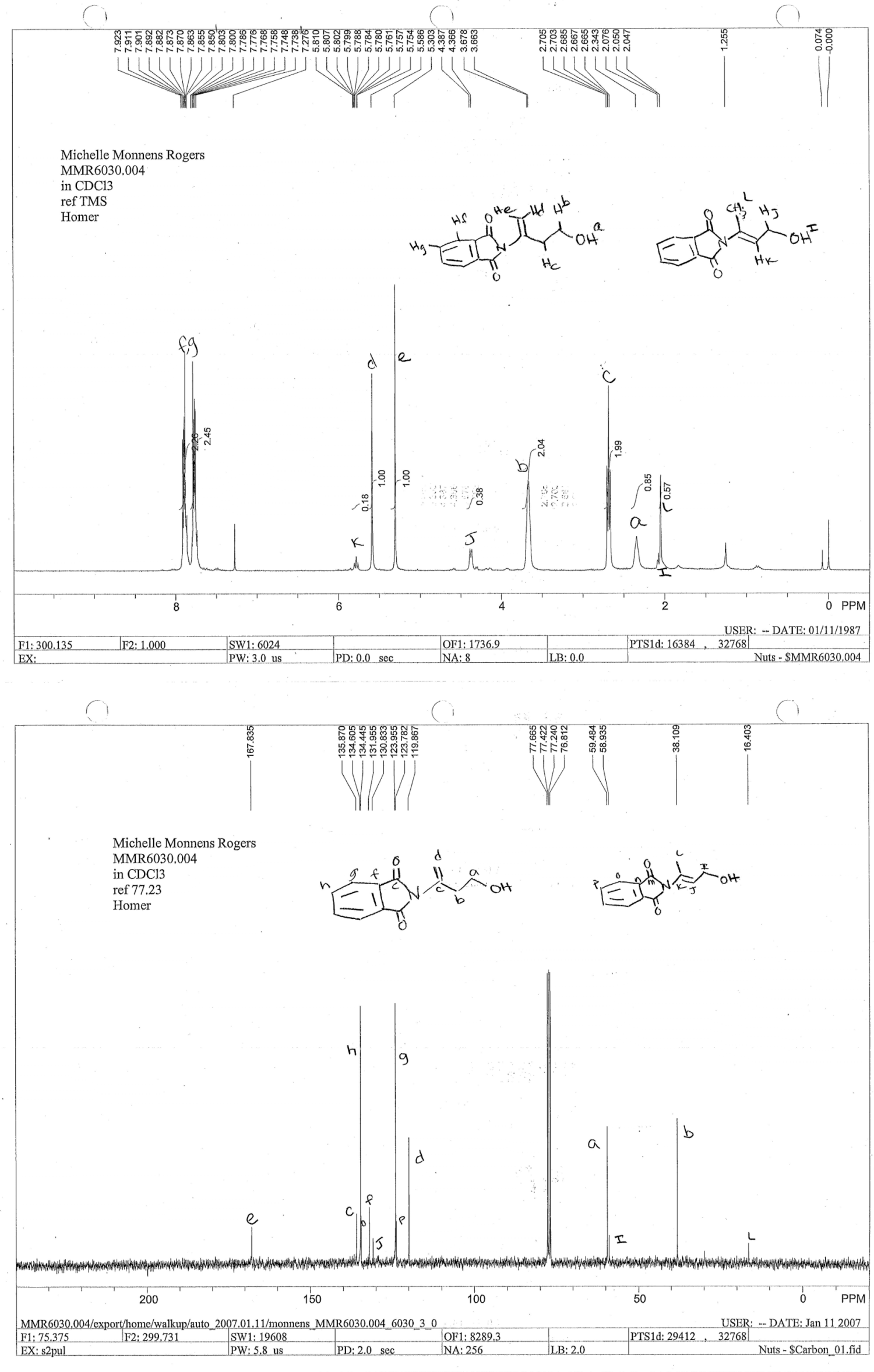

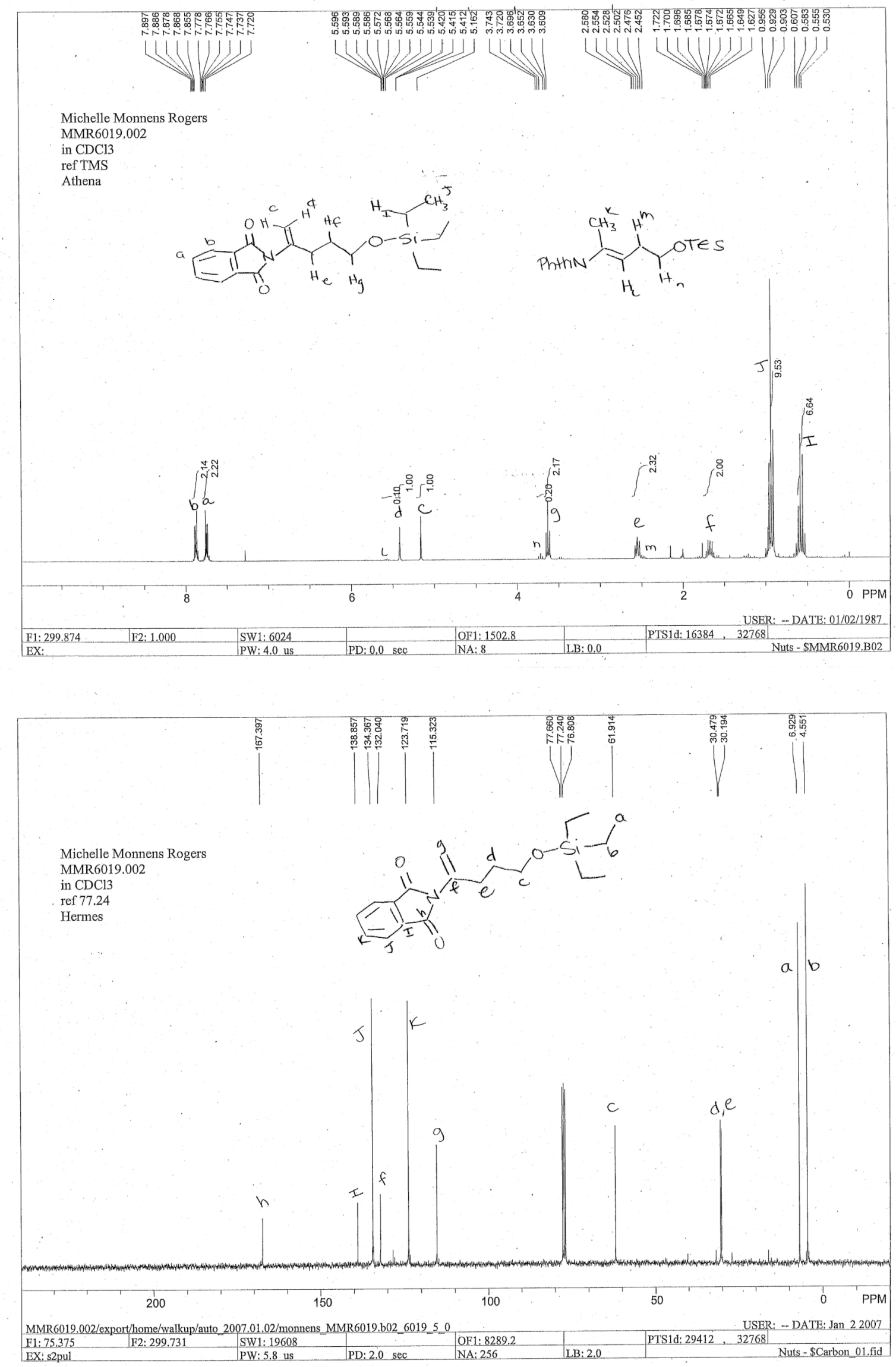

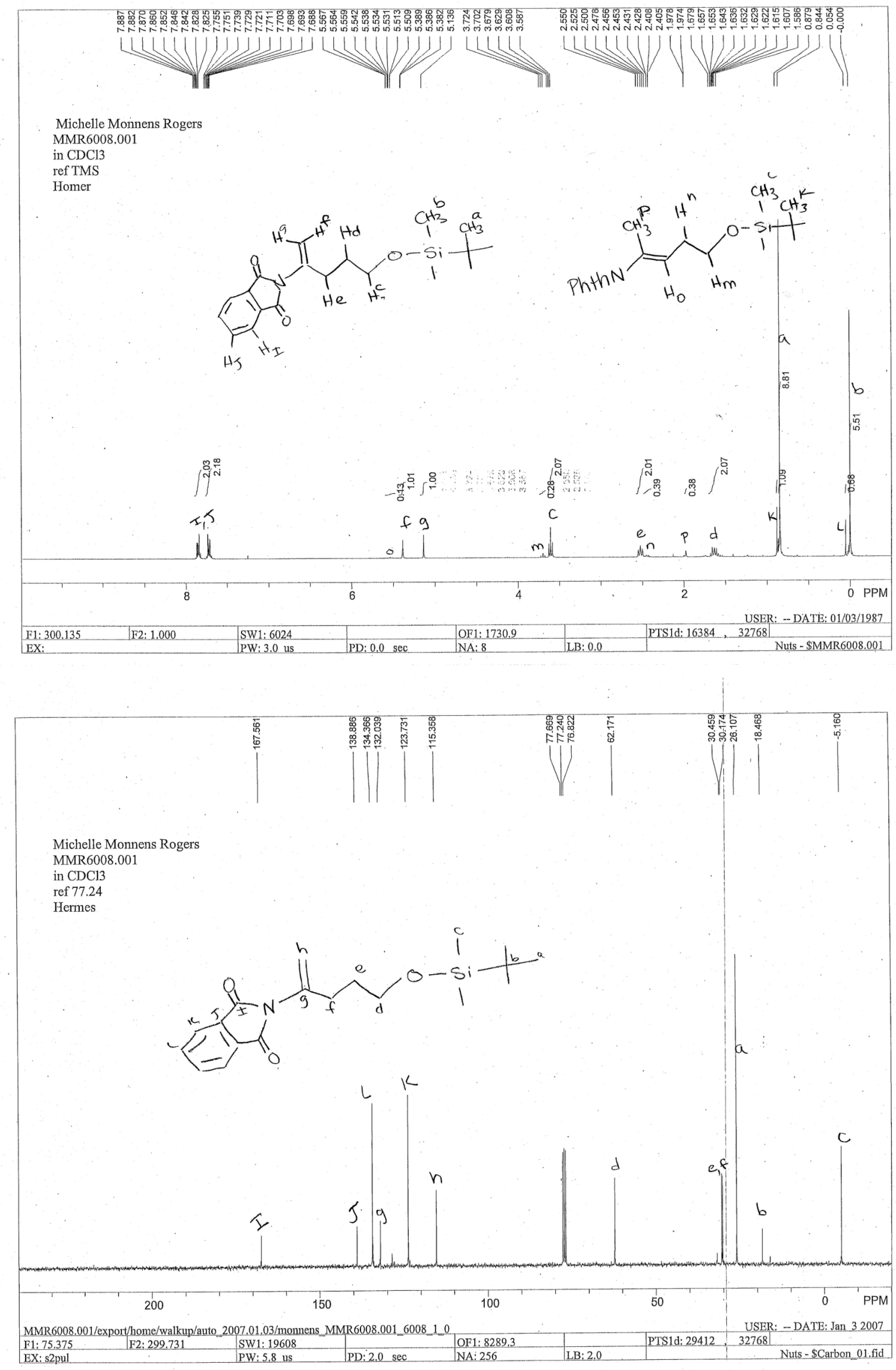

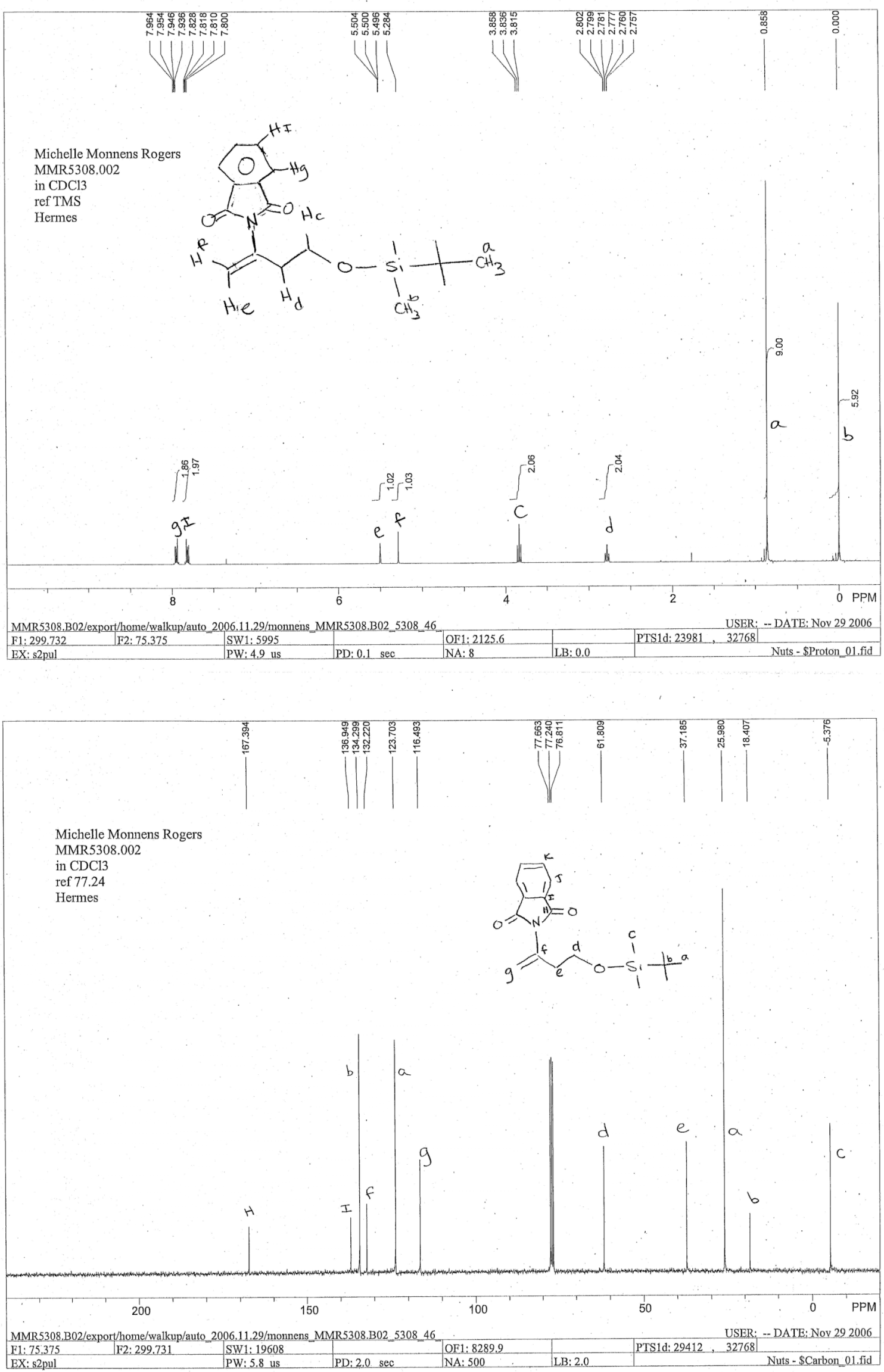

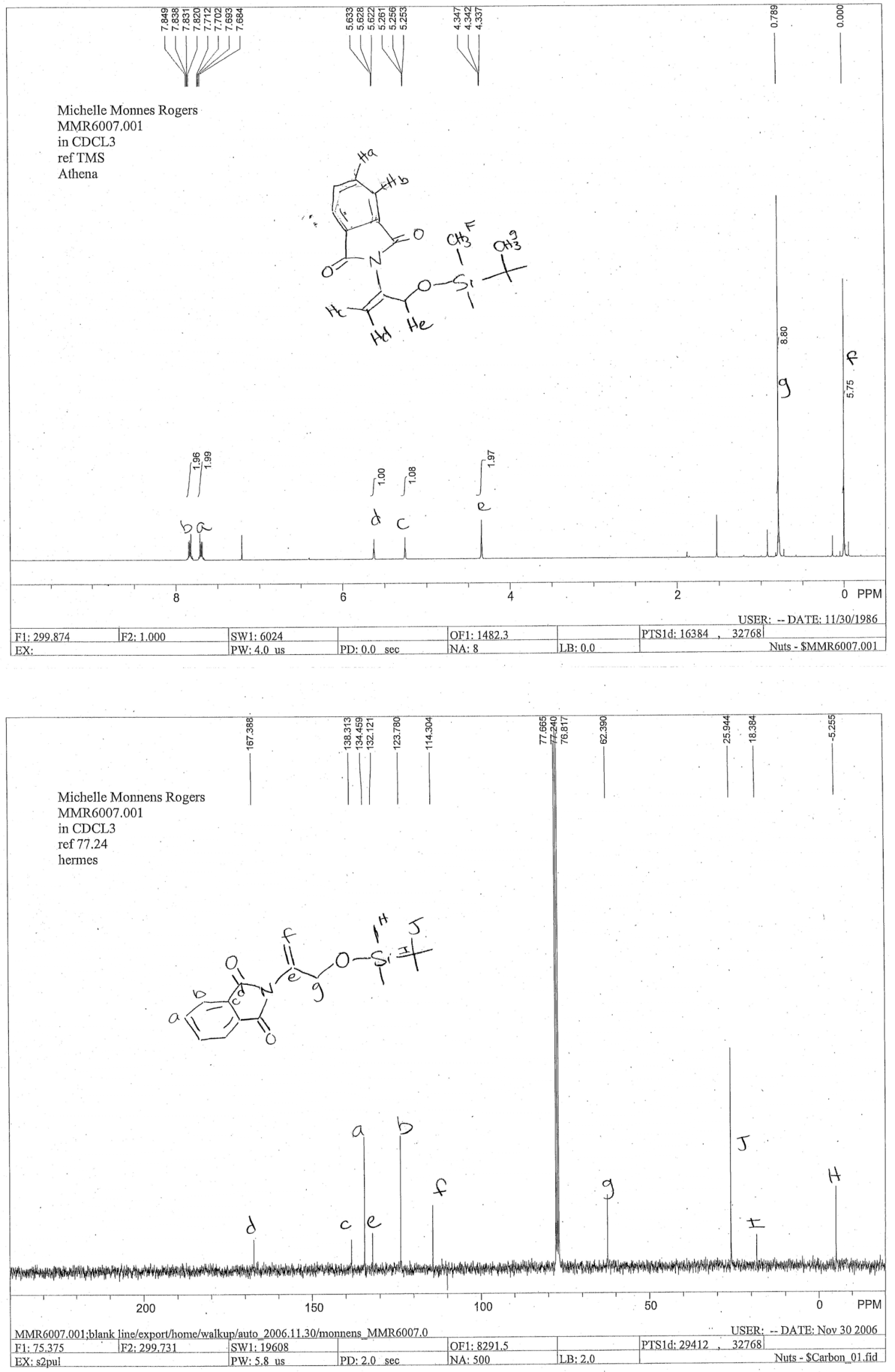